\title{
A Study of Disorder Shell Effects on the Mechanical Properties of SiC Nanowires
}

\author{
Bin Zheng* - Huiling Du \\ 1Xi'an University of Science and Technology, School of Materials Science and Engineering, China
}

The mechanical properties of SiC nanowires were investigated using molecular dynamics simulation method. The results show that the disorder shell layer reduced the elastic modulus of SiC nanowires. This reduction mainly depends on the thickness and the atomic type of the disorder shell. Thicker $\mathrm{C}$ and Si disorder layers can strengthen and weaken the nanowires, respectively. Also, the core-shell wires have sizedependent strength, which can be understood by examining the variation of Young's modulus and the volume fraction of the isolated core and isolated shell. Furthermore, the disorder coating was found to facilitate the brittle-ductile transition in the SiC core. The simulation results are expected to help the design and manufacturing of complex nanoscale architectures with desired mechanical properties.

Keywords: core/shell nanowire, mechanical properties, Young's modulus, molecular dynamics simulation

Highlights

- The disorder shell layer reduces the elastic modulus of SiC nanowires.

- $\quad$ Thicker $C$ and Si disorder layers can strengthen and weaken the nanowires, respectively.

- $\quad$ The bigger the SiC core, the larger Young's modulus of the core-shell nanowires.

- $\quad$ The modulus of the crystal core dominates the strength of the whole wire.

- The disorder shell induces the amorphization of the crystal core and makes the semiconductor core-shell nanowires ductile.

\section{INTRODUCTION}

The core-shell heterostructure of nanomaterials is critical to the extraordinary tunability of the properties of nanomaterials [1]. The crystalline phase structure has been the subject of many experimental investigations. More recently, crystalline coredisorder shell materials have attracted attention, owing to their interface boundary effect applied in lithium ion batteries and high-conversion energy solar cell devices [2] and [3].

The application performance of crystallinedisorder core-shell materials depends on their stable mechanical properties. Recently, there has been considerable interest in studying the effect of the core-shell interface on the mechanical properties of nanowires (NWs) [4] and [5]. Some investigations have shown that high internal stress gradients, owing to lattice mismatch, develop at the interface, which then results in an unusual deformation mechanism in metallic nanomaterials [6]. For brittle semiconductor materials, the disorder shell is considered to play a significant role in stabilizing the crystalline core [7] and [8] and in dominating the brittle-ductile (BD) transition [9]. Therefore, the core-shell structure can modulate the elastic modulus of NWs and induce plastic deformation. Despite the progress in understanding the mechanical deformation of coreshell NWs, knowledge of the tuneable properties of such nano-hetero-structures remains limited.
In this paper, we focused on the mechanical properties of core-shell NWs with silicon carbide $(\mathrm{SiC})$ crystalline core and disorder carbon $(d-\mathrm{C})$ or silicon $(d-\mathrm{Si})$ shell, which have been synthesized experimentally by many routes [10] and [11]. We performed calculations for the $\mathrm{SiC}-d-\mathrm{C}$ and $\mathrm{SiC}-$ $d$-Si core-shell NWs to evaluate their mechanical properties and to understand the core-shell interface effect.

\section{SIMULATION MODEL}

The simulation model consists of the [111]-oriented $3 \mathrm{C}-\mathrm{SiC}$ crystalline core and the disorder layer. We built the atomic model of the $\mathrm{SiC}$ core with a hexagonal cross section (Fig. 1). The size of the nanowire cores ( $1 \mathrm{~nm}, 1.3 \mathrm{~nm}$, and $1.7 \mathrm{~nm}$ diameters) is defined as the average from the centre to the corner and edge distances. To obtain the disorder shell, crystalline $\mathrm{C}$ and $\mathrm{Si}$ diamond specimens were heated above their melting points (C: $3820 \mathrm{~K}$ and Si: 2500 $\mathrm{K}$ ), and then cooled to room temperature at $200 \mathrm{~K} /$ picosecond (ps) to obtain disorder bulk materials for cutting the hexagonal disorder shells [12] and [13]. Subsequently, core-shell NWs were assembled (Fig. 1) by inserting the crystalline core into disorder shells with thicknesses of $0.3 \mathrm{~nm}, 0.6 \mathrm{~nm}$, and $0.9 \mathrm{~nm}$. An initial distance of $0.15 \mathrm{~nm}$ between core and shell was designed for interface structure relaxation. 
Molecular dynamics (MD) simulations were used to perform uniaxial deformation under simulated quasistatic conditions and to obtain the mechanical properties of SiC-disorder core-shell NWs. The LAMMPS software [14] with a time step of 1 femtosecond ( $\mathrm{fs}$ ) was used to carry out the MD simulations. Before loading, two order steps were performed to establish the atomic rearrangement of the free surface or the core-shell interface. First, energy minimization based on the conjugate gradient (CG) algorithm was used to guarantee the atomic optimization position at zero temperature. Second, the internal stresses of the initial nanostructure were released by relaxation under constant atmosphere pressure for $1 \mathrm{~ns}$ and constant volume for another $1 \mathrm{~ns}$ to thermally equilibrate the system at $300 \mathrm{~K}$ using a Nosé-Hoover thermostat [15] and [16]. In a stepwise manner, the compressive force was loaded along the axial direction with periodic boundary conditions. For each step, the uniform displacement increment was $0.1 \mathrm{~nm}$ and the relaxation time at constant temperature was 500 ps. An equivalent 0.2 $\mathrm{m} / \mathrm{s}\left(0.002 \% \mathrm{ps}^{-1}\right)$ compressive rate was obtained. No significant difference appeared when the compressive rate decreased to $0.1 \mathrm{~m} / \mathrm{s}\left(0.001 \% \mathrm{ps}^{-1}\right)$. All the reported numerical simulations were performed using the Tersoff potential for $\mathrm{C}, \mathrm{Si}$, and $\mathrm{SiC}$, where the total energy of the system with one-body, two-body, and three-body contributions and testing indicated its transferability from the molecular to the bulk regime, making it a good choice for atomistic simulations [17].

(a)
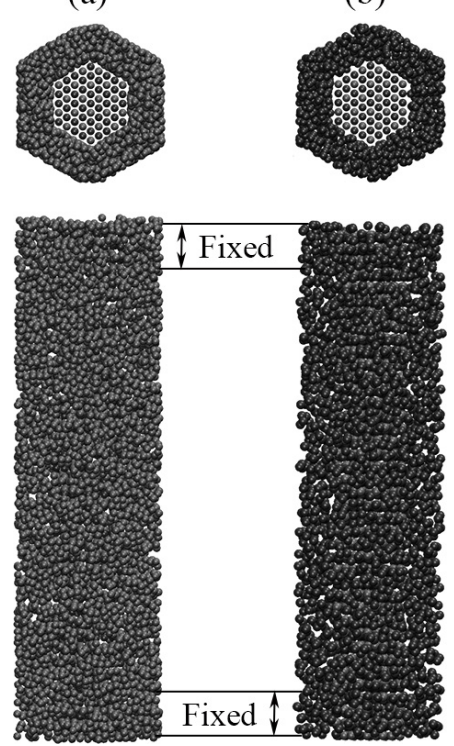

Fig. 1. Top and side view of a) SiC-d-C and b) SiC-d-Si core-shell nanowire
In the simulations, uniaxial loading was applied along the $\mathrm{z}$ direction. The reported stresses $\left(\sigma^{z z}\right)$ were calculated by summing the stress tensor $\left(f_{t}^{z}\right)$ of all atoms along the $z$-direction and then dividing them by the volume of the system ( $V$ ), as shown in Eq. (1). The volume $V$ is the product of the hexagonal crosssectional area and the equilibrium length in every loading step. For strain from $-3.0 \%$ to $3.0 \%$, linear regression, and the stress-strain curves were used to estimate Young's modulus $(Y)$ with Eq. (2).

$$
\begin{gathered}
\sigma^{z z}=\frac{1}{V} \sum_{i}^{N} f_{i}^{z}, \\
\sigma^{z z}=Y \varepsilon_{z} .
\end{gathered}
$$

\section{RESULTS AND DISCUSSION}

The stress-strain curves for SiC-disorder core-shell nanowire materials loaded both in compression and tension are shown in Fig. 2, from which we find the typical characteristics of the mechanical properties of semiconductor nanowires: ultra-strength and super ductility. Their elastic strains are larger than $3 \%$, and failure strength reaches to above $10 \mathrm{GPa}$ for all nanowires. Also, Fig. 2 shows the tuneable mechanical properties of the nanowires through the thickness and the type of shell layer.

Young's modulus can be extracted from the stress-strain curves, as shown in Table 1. The elastic strength of wires was compared with the different sizes of core and shell. Furthermore, the Si core was employed to show the effect of the disorder shell on Young's modulus of core/shell NWs.

From Table 1, it is clear that Young's modulus of naked NWs is bigger than that of wires coated with disorder layers; this observation is consistent with previous reports [18].

Previous publications [1] showed that the high internal stress gradients, owing to lattice mismatch, exist at the core/shell interface, which can change the mechanical properties of the wires, although the shell layers are crystallized structures. In this work, the shell layer becomes a disordered structure, which potentially will saturate those dangling bonds on the core surface due to no limitation of the lattice. The atomic stress distribution will be analyzed to understand the strength of the wires coated by the disorder shell, as shown in Fig. 3.

From Fig. 3a, we found that most of the atoms in naked NW are under compressive stress state (peak 1). All those atoms located on the surface of the nanowire 

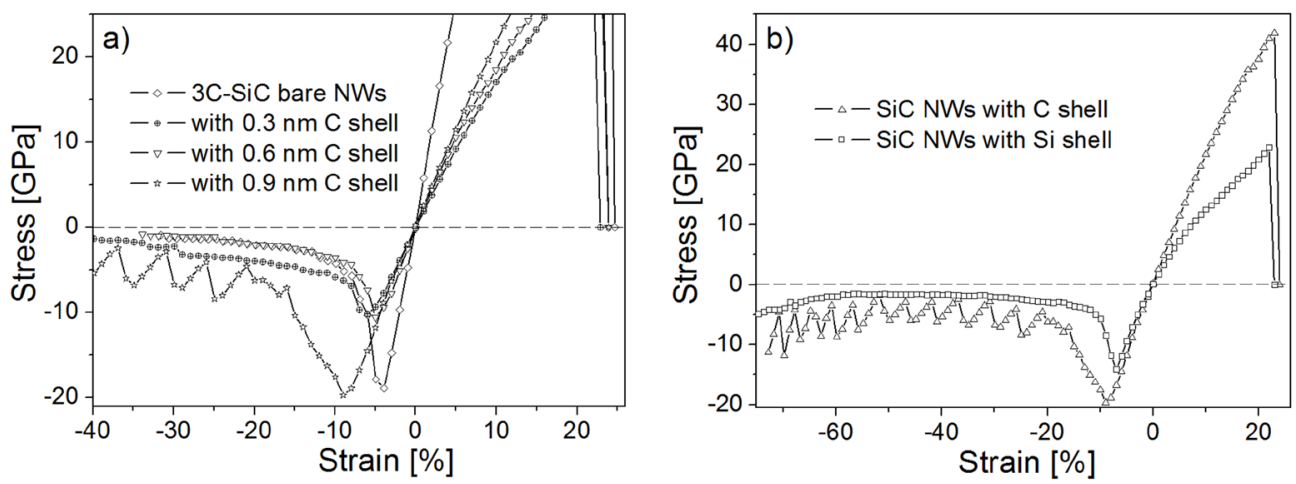

Fig. 2. Stress vs. strain curves for a) $3 C-S i C$ nanowires with different thicknesses of disorder carbon shell and b) the SiC nanowires coated with different types of disorder shell

Table 1. Young's modulus of crystalline-disorder core-shell nanowires with different core diameters and shell thicknesses; the values were obtained using linear regression from the stress-strain curves for strain from $-3.0 \%$ to $3.0 \%$

\begin{tabular}{|c|c|c|c|c|c|c|c|c|c|c|}
\hline \multirow[b]{3}{*}{$\begin{array}{c}\text { Shell size } \\
{[\mathrm{nm}]}\end{array}$} & \multicolumn{10}{|c|}{ Young's modulus (GPa) of the core/shell NWs } \\
\hline & \multicolumn{2}{|c|}{ SiC core, $D=1 \mathrm{~nm}$} & \multicolumn{2}{|c|}{ SiC core, $D=1.3 \mathrm{~nm}$} & \multicolumn{2}{|c|}{ SiC core, $D=1.7 \mathrm{~nm}$} & \multicolumn{2}{|c|}{ SiC core, $D=2 \mathrm{~nm}$} & \multicolumn{2}{|c|}{ Si core, $D=4.2 \mathrm{~nm}$} \\
\hline & $\begin{array}{l}d-C \\
\text { shell }\end{array}$ & $\begin{array}{l}d \text {-Si } \\
\text { shell }\end{array}$ & $\begin{array}{l}d-C \\
\text { shell }\end{array}$ & $\begin{array}{l}d \text {-Si } \\
\text { shell }\end{array}$ & $\begin{array}{l}d-C \\
\text { shell }\end{array}$ & $\begin{array}{l}d-\mathrm{Si} \\
\text { shell }\end{array}$ & $\begin{array}{c}\text { Shell size } \\
{[\mathrm{nm}]}\end{array}$ & $\begin{array}{l}d \text {-SiC } \\
\text { shell }\end{array}$ & $\begin{array}{c}\text { Shell size } \\
{[\mathrm{nm}]}\end{array}$ & $\begin{array}{l}d-\mathrm{Si} \\
\text { shell }\end{array}$ \\
\hline 0 & 561 & 561 & 547 & 547 & 544 & 544 & 0 & 520 & 0 & 131.1 \\
\hline 0.3 & 250 & 209 & 269 & 259 & 276 & 248 & 0.3 & 440 & 0.3 & 113.9 \\
\hline 0.6 & 258 & 94 & 272 & 189 & 288 & 218 & 0.6 & 435 & 0.6 & 103.6 \\
\hline 0.9 & 259 & 92 & 273 & 151 & 292 & 173 & 0.9 & 430 & 0.9 & 82.7 \\
\hline
\end{tabular}

a) Naked SiC NW

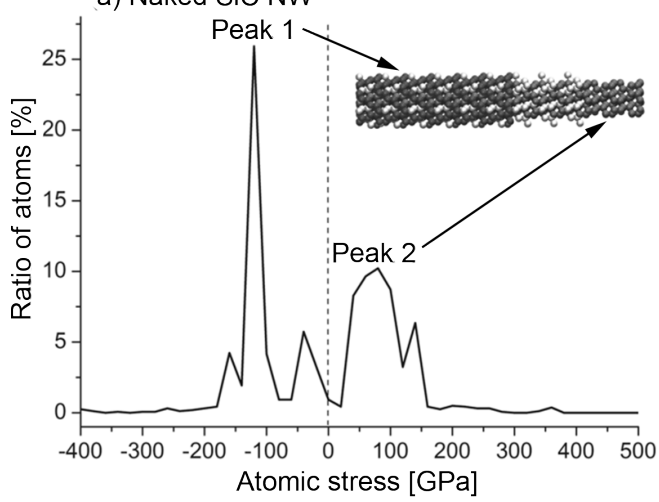

c) Disorder shell in core/shell NW

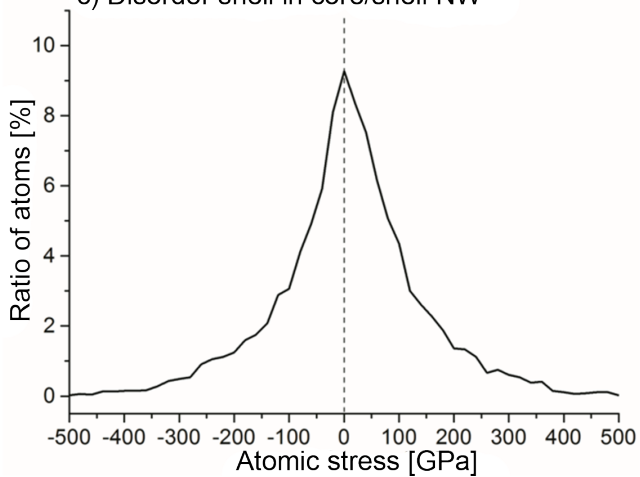

b) $\mathrm{SiC}$ core in core/shell NW

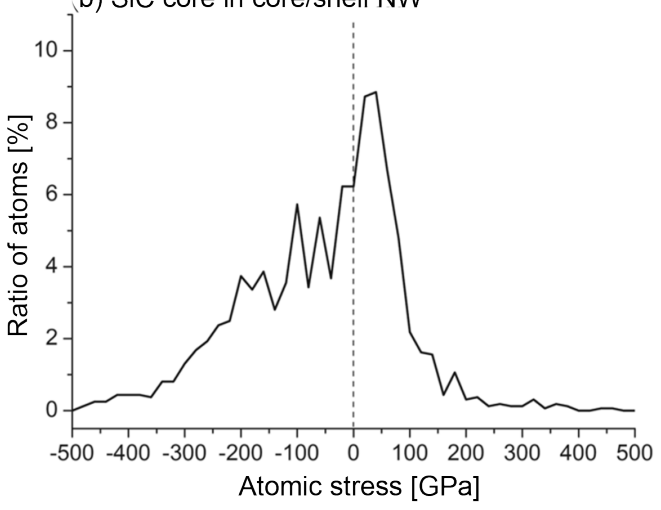

d) $\mathrm{SiC} / \mathrm{d}-\mathrm{C}$ core/shell NW

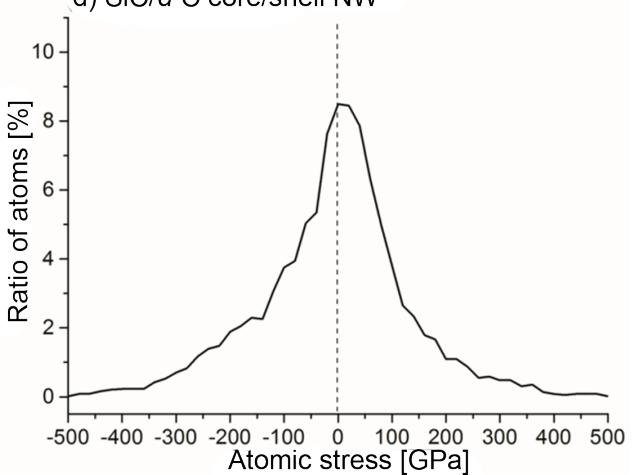

Fig. 3. Atomic stress distribution in a) naked SiC nanowire, b) SiC core, c) disorder $\mathrm{C}$ shell and d) the whole core/shell nanowire; the strain of all nanowires is $0 \%$ 
Table 2. Young's modulus and volume fraction of core-shell nanowires

\begin{tabular}{|c|c|c|c|c|c|c|c|c|c|}
\hline \multirow[b]{2}{*}{ Core size [nm] } & \multicolumn{3}{|c|}{$0.9 \mathrm{~nm} d-\mathrm{C}$ shell } & \multicolumn{3}{|c|}{$0.6 \mathrm{~nm} d-\mathrm{C}$ shell } & \multicolumn{3}{|c|}{$0.3 \mathrm{~nm} d-\mathrm{C}$ shell } \\
\hline & 1 & 1.3 & 1.7 & 1 & 1.3 & 1.7 & 1 & 1.3 & 1.7 \\
\hline$Y$ [GPa] of core/shell & 259 & 273 & 292 & 258 & 272 & 288 & 250 & 269 & 276 \\
\hline$Y[\mathrm{GPa}]$ of core & 426 & 441 & 449 & 415 & 445 & 461 & 446 & 457 & 471 \\
\hline$V_{\text {core }} / V_{\text {core-shell }}[\%]$ & 14 & 19 & 24 & 23 & 27 & 33 & 40 & 44 & 46 \\
\hline$Y[\mathrm{GPa}]$ of shell & 231 & 235 & 244 & 211 & 212 & 204 & 168 & 124 & 113 \\
\hline$V_{\text {shell }} / V_{\text {core-shell }}[\%]$ & 86 & 81 & 76 & 77 & 73 & 67 & 60 & 56 & 54 \\
\hline
\end{tabular}

and the surface dangling bonds result in their high compressive state. To reach the force equilibrium of the whole wire, the atoms in the inner region are under a tension stress state (peak 2 in Fig. 3a). When stretching the naked NWs, the external force has to overcome an additional barrier coming from the surface atoms in a compressive state. Furthermore, the compressive loading will be resisted by the inner atoms under tension. The high fraction of surface atoms in nanowires guarantees above non-uniform stress distribution and then strengthen the whole wire. This is especially true in thinner nanowires, i.e. size effect.

The disorder shell can change the stress distribution of the $\mathrm{SiC}$ core, reflecting the fact that most of the atoms in the wire are close to the zero stress state (Fig. 3b). The shell atoms at the interface saturate those dangling bonds in the surface of the $\mathrm{SiC}$ core and then the surface stress in the naked state is almost released. In addition, we observed that most of the atoms in the disorder shell are in a zero stress state (Fig. 3c), regardless of the existence of a free surface. This can be understood by the disorder structure, in which atoms can freely choose their coordination atoms to eliminate the surface stress. Therefore, most of the atoms in SiC-disorder core-shell nanowires are in near a zero stress state (Fig. 3d), and then the wires are softer than their naked counterparts.

Another interesting trend in Table 1 is that SiC$d$-C and SiC- $d$-Si NWs stiffen because of the large crystalline core, for fixed disorder shell thickness. In order to understand this size effect, we rearrange the calculation equation of Young's modulus of the coreshell NWs, as shown below.

According to Eq. (1), the deformation stresses of the core-shell NWs under loading are:

$$
\begin{aligned}
\sigma_{\text {core-shell }}^{z z} & =\frac{1}{V_{\text {core-shell }}}\left(\sum_{i=1}^{N_{\text {core }}} f_{i}^{z}+\sum_{i=N_{\text {core }}+1}^{N} f_{i}^{z}\right)= \\
& =\frac{V_{\text {core }}}{V_{\text {core-shell }}} \sigma_{\text {core }}^{\mathrm{zz}}+\frac{V_{\text {shell }}}{V_{\text {core-shell }}} \sigma_{\text {shell }}^{\mathrm{zz}} .
\end{aligned}
$$

We then obtained the Young's modulus of the core-shell NWs by integrating Eqs. (2) and (3) [19].

$$
Y_{\text {core-shell }}=\frac{V_{\text {core }}}{V_{\text {core-shell }}} Y_{\text {core }}+\frac{V_{\text {shell }}}{V_{\text {core-shell }}} Y_{\text {shell }} \text {. }
$$

From the above formula, we see that the Young's modulus of core-shell NWs is the weighted combination of the Young's modulus of the cores and

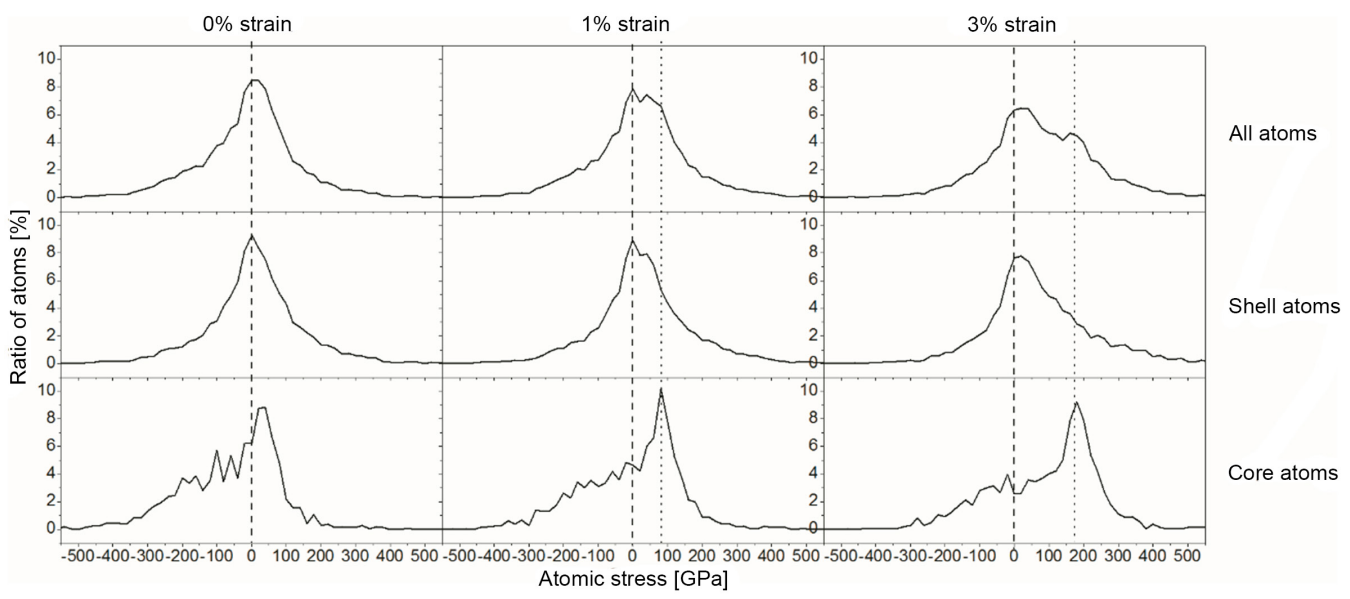

Fig. 4. Atomic stress distribution in SiC-d-C core-shell nanowires with different strains 
the shells. The weight factor is the fractional volume $V_{\text {core }} / V_{\text {core-shell }}\left(V_{\text {shell }} / V_{\text {core-shell }}=1-V_{\text {core }} / V_{\text {core-shell }}\right)$. These values are represented in Table 2. From this table, we found that both of the modulus values of the core and the shell increases with the rising of the core size. Moreover, the Young's modulus of the core is 2 to 3 times larger than that of the shell. Although the value of $V_{\text {shell }} / V_{\text {core-shell }}$ becomes small in the large core, the whole nanowires still stiffen due to the more important role of the core in deciding the strength of the core/shell wires. The role of the core and the shell in the deformation of core-shell NWs is confirmed by the atomic stress distribution of wires under loading (Fig. 4). This figure shows that most of the atoms in core enter a tensile stress state in respond to the external stretching force (peak right shift). Comparatively, the peak of atoms fraction in shell still locates in the zero stress position during loading.

The volumes in Table 2 are nominal values, and we ignored the temperature and core-shell interface space. The latter can cause difficulties in defining the volumes of the isolated cores and shells in coreshell NWs. Liu et al. [19] defined the geometric and physical volumes. The physical volume is due to the existence of the core-shell interface region. The basis for this definition is the division of the interface region into two parts that belong to the core and the shell. Liu et al. [19] also calculated the physical volume according to the ratio of the bond lengths of the core and the shell. In the proposed core-shell model, the ratio of bond lengths was not obtained owing to the disordered structure. Instead, we used the ratio of the average atomic potential energy of the core and the shell to divide the interface region and then calculated the physical volume as well as the Young's modulus of the isolated core and the isolated shell, as shown in Tables 2 and 3.

For fixed crystalline core diameter, the Young's modulus of $\mathrm{SiC}-d-\mathrm{Si}, \mathrm{SiC}-d-\mathrm{SiC}$, and $\mathrm{Si}-d-\mathrm{Si}$ core/ shell NWs decreases as the thickness of the disorder shell increases (Table 1). An opposite trend was observed in SiC- $d$-C core/shell NWs, i.e. the thicker the disorder carbon shell is, the larger the modulus. This difference can be understood by comparing the strength of disorder carbon and disorder silicon. In disorder carbon, we do find some ultra-strong local structures (diamond-like carbon), which results in the relatively high strength in SiC- $d$-C core/shell NWs.

In addition to affecting the strength, the disorder coating can cause plastic deformation in semiconductor materials, as shown in Fig. 5, from which it can be seen that NWs with or without disorder coating buckle under uniaxial compression.
Makeev et al. [20] also reported the buckling modes of $\mathrm{SiC}$ NWs. Buckling is related to the smaller diameter for a given length of nanowires regardless of the core-shell structure. No structural defects appeared in naked $\mathrm{SiC}$ wires below the critical strain (31\%) with additional compression. Upon exceeding the critical strain, the abrupt rupture of the $\mathrm{Si}-\mathrm{C}$ bond outside the buckling region and the subsequent clean cut indicates brittle behaviour. In contrast, for core-shell NWs, no clean rupture was observed in the isolated core and shell during deformation, as indicated by the plastic deformation of $\mathrm{SiC} \mathrm{NWs} \mathrm{owing} \mathrm{to} \mathrm{the} \mathrm{disorder} \mathrm{layer.}$ Comparing the configuration of the naked wire and the isolated core in Fig. 5, we see that the structure of the latter is more disordered. We believe that the amorphization of the core induced by the disorder shell is the main contributor to the ductile properties of core-shell NWs.

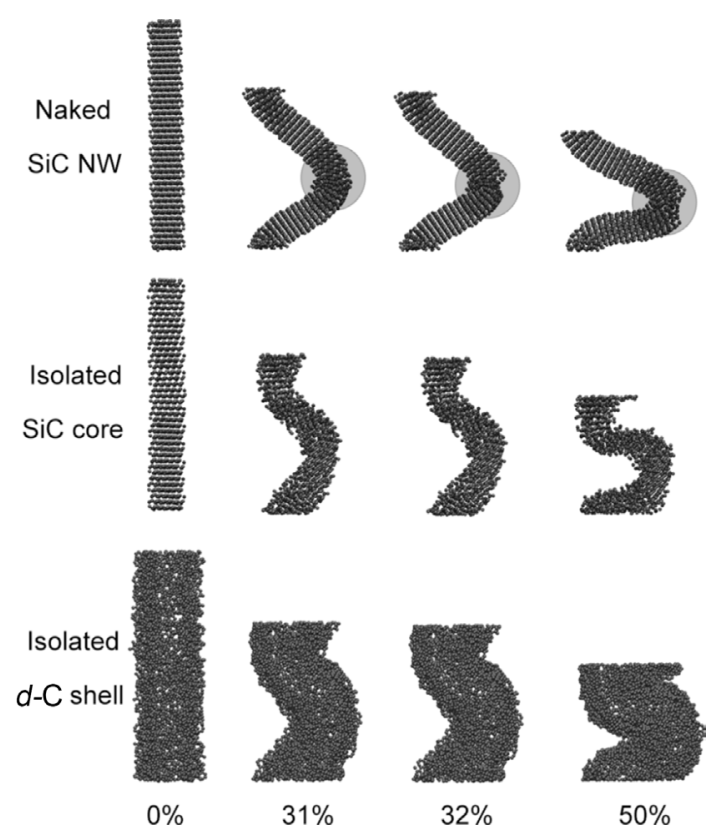

Fig. 5. Atomic configurations of selected stages of naked SiC nanowires with $1.3 \mathrm{~nm}$ diameter, isolated SiC core with $1.3 \mathrm{~nm}$ diameter, and a disorder layer with $0.6 \mathrm{~nm}$ thickness

\section{CONCLUSION}

We used molecular dynamics to simulate the mechanical deformation of $\mathrm{SiC}-d-\mathrm{C}$ and $\mathrm{SiC}-d-\mathrm{Si}$ core-shell nanowires and studied the Young's modulus and plastic deformation due to the disorder shell. We found that the strength of $\mathrm{SiC}$ nanowires decreased after the application of disorder coating, as can be attributed to the release of the interface atomic stress by the disorder shell. Furthermore, the size effect was 
observed, i.e. the bigger the $\mathrm{SiC}$ core, the larger the Young's modulus of the core-shell nanowires. The interface effect was considered to divide the core-shell interface into two parts belonging to the core and the shell, and the modulus of the crystal core dominates the strength of the whole wire. Additional deformation beyond the elastic region suggests that the disorder shell induces the amorphization of the crystal core and makes the semiconductor core-shell nanowires ductile. The simulation showed that the mechanical properties of the semiconductor core-shell nanowires depend on the type and thickness of the disorder shell, which will facilitate their application to nanoscale devices.

\section{ACKNOWLEDGEMENTS}

This work was supported by the Natural Science Foundation of China under grant 21503165 and 51372197, the Key Innovation Team of Shaanxi Province (2014KCT-04), the Major International Joint Research Program of Shaanxi Province (2012KW-10), and the Science and Technology Program of Shaanxi Province (2013KJXX-42).

\section{REFERENCES}

[1] Zheng, B., Wang, Y.N., Qi, M., Williams, E.H. (2009). Phase boundary effects on the mechanical deformation of core/shell $\mathrm{Cu} / \mathrm{Ag}$ nanoparticles. Journal of Materials Research, vol. 24, no. 7, p. 2210-2214, DOI:10.1557/jmr.2009.0263.

[2] Chen, H., Xu, J., Chen, P.C., Fang, X., Qiu, J., Fu, Y., Zhou, C. (2011). Bulk synthesis of crystalline and crystalline core/ amorphous shell silicon nanowires and their application for energy storage. ACS Nano, vol. 5, no. 10, p. 8383-8390, DOI:10.1021/nn203166w.

[3] Li, Z.Q., Li, X.D., Liu, Q.Q., Chen, X.H., Sun, Z., Liu, C., Ye, X.J., Huang, S.M. (2012). Core/shell structured NaYF4:Yb3+/Er3+/ $\mathrm{Gd}+3$ nanorods with $\mathrm{Au}$ nanoparticles or shells for flexible amorphous silicon solar cells. Nanotechnology, vol. 23, no. 2, paper 025402, D0I:10.1088/0957-4484/23/2/025402.

[4] Khachadorian, S., Papagelis, K., Ogata, K., Hofmann, S., Phillips, M.R., Thomsen, C. (2013). Elastic properties of crystalline-amorphous core-shell silicon nanowires. The Journal of Physical Chemistry C, vol. 117, no. 8, p. 4219-4226, DOI:10.1021/jp309842c.

[5] Sandmann, B., Happ, B., Perevyazko, I., Rudolph, T., Schacher, F.H., Hoeppener, S., Mansfeld, U., Hager, M.D., Fischer, U.K., Burtscher, P., Moszner, N., Schubert, U.S. (2015). Incorporation of core-shell particles into methacrylate based composites for improvement of the mechanical properties. Polymer Chemistry, vol. 6, no. 29, p. 5273-5280, D0I:10.1039/C4PY01544D.

[6] Fang, Q.H., Liu, Y.W., Jin, B., Wen, P.H. (2009). Interaction between a dislocation and a core-shell nanowire with interface effects. International Journal of Solids and Structures, vol. 46, no. 6, p. 1539-1546, D0l:10.1016/j.jjsolstr.2008.11.026.

[7] Yang, Z.X., Jun, Y.J., Zhu, F., Zhang, Y. (2005). Helical nanocables with $\mathrm{SiC}$ core and $\mathrm{SiO}_{2}$ shell. Physica $\mathrm{E}$, vol. 25, no. 4, p. 395-398, D0l:10.1016/j.physe.2004.07.006.

[8] Zhang, H.F., Wang, C.M., Wang L.S. (2002). Helical crystalline $\mathrm{SiC} / \mathrm{SiO}_{2}$ core-shell nanowires. Nano Letter, vol. 2, no. 9, p. 941-944, DOI:10.1021/nl025667t.

[9] Dai, L., Sow, C.H., Lim, C.T., Cheong, W.C.D., Tan V.B.C. (2009). Numerical investigations into the tensile behavior of $\mathrm{TiO} 2$ nanowires: Structural deformation, mechanical properties, and size effects. Nano Letter, vol. 9, no. 2, p. 576-582, DOI:10.1021/nl8027284.

[10] Shen, G., Chen, D., Tang, K., Qian, Y., Zhang, S. (2003). Silicon carbide hollow nanospheres, nanowires and coaxial Nanowires. Chemical Physics Letters, vol. 375, no. 1-2, p. 177 184, DOl:10.1016/S0009-2614(03)00877-7.

[11] Yang, G.Z., Cui, H., Sun, Y., Gong, L., Chen, J., Jiang, D., Wang, C.X. (2009). Simple catalyst-free method to the synthesis of $\beta$-SiC nanowires and their field emission properties. The Journal of Physical Chemistry C, vol. 113, no. 36, p. 1596915973, DOl:10.1021/.jp906167s.

[12] Lu, Q., Marks, N., Schatz, G.C., Belytschko, T. (2008). Nanoscale fracture of tetrahedral amorphous carbon by molecular dynamics: Flaw size insensitivity. Physical Review B, vol. 77, no. 1, p. 014109, D0l: 10.1103/PhysRevB.77.014109.

[13] Mathioudakis, C., Kopidakis, G., Kelires, P. C., Wang, C.Z., Ho, K.M. (2004). Physical trends in amorphous carbon: A tightbinding molecular-dynamics study. Physical Review B, vol. 70, no. 12, p. 125202, Dol:10.1103/PhysRevB.70.125202.

[14] Plimpton, S. (1995). Fast parallel algorithms for short-range molecular-dynamics. Journal of Computational Physics, vol. 117, no. 1, p. 1-19, D0I:10.1006/jcph.1995.1039.

[15] Hoover, W.G. (1985). Canonical dynamics: Equilibrium phasespace distributions. Physical Review A, vol. 31, no. 3, p. 16951697, D0l:10.1103/PhysRevA.31.1695.

[16] Nosé, S. (1984). A unified formulation of the constant temperature molecular-dynamics methods. The Journal of Chemical Physics, vol. 81, no. 1, p. 511-519, DOI:10.1063/1.447334.

[17] Erhart, P., Albe, K. (2005). Analytical potential for atomistic simulations of silicon, carbon, and silicon carbide. Physical Review B, vol. 71, no. 3, p. 035211, Dol:10.1103/ PhysRevB.71.035211.

[18] Jing, Y., Meng, Q. (2010). Molecular dynamics simulations of the mechanical properties of crystalline/amorphous silicon core/shell nanowires. Physica B, vol. 405, no. 10, p. 24132417, D0I:10.1016/J.physb.2010.02.056.

[19] Liu, X.W., Hu, J., Pan, B.C. (2008). The composition-dependent mechanical properties of $\mathrm{Ge} / \mathrm{Si}$ core-shell nanowires, Physica E, vol. 40, no. 10, p. 3042-3048, Dol:10.1016/j. physe.2008.03.011.

[20] Makeev, M.A., Srivastava, D., Menon, M. (2006). Silicon carbide nanowires under external loads: An atomistic simulation study. Physical Review B, vol. 74, no. 16, p. 165303, DOI:10.1103/PhysRevB.74.165303. 